\title{
The impact of MC-ICP-MS plasma conditions on the accuracy and precision of stable isotope measurements evaluated for barium isotopes
}

\author{
YANG YU' ${ }^{\prime}$, CHRISTOPHER SIEBERT' ${ }^{\prime}$ JAN FIETZKE', \\ TYLER GOEPFERT ${ }^{2}$, ED HATHORNE', ZHIMIAN CAO ${ }^{3}$ AND \\ MARTIN FRANK' \\ GEOMAR Helmholtz Centre for Ocean Research Kiel, \\ Wischhofstraße 1-3, Kiel 24148, Germany \\ ${ }_{2}$ School of Earth and Space Exploration, Arizona State \\ University, Tempe, AZ 85287, USA \\ ${ }^{3}$ State Key Laboratory of Marine Environmental Science \\ and College of Ocean and Earth Sciences, Xiamen \\ University, Xiamen 361102, China
}

Stable barium isotope measurements with MC-ICP-MS remain an analytical challenge and can be considerably affected by the presence of matrix elements, even when applying double spiking. Therefore significant efforts were invested in previous studies to develop efficient barium purification methods. However, due to the high variability in matrix/barium ratios for diverse sample matrices, potential matrix effects can still not be excluded. While a lot of effort has been invested into improving the chemical separation protocols, the impact of plasma conditions on the accuracy and precision of stable isotope measurements has rarely been considered. Here we present a systematic investigation of the relationship between plasma conditions, instrumental mass fractionation (IMF) and impurity (i.e. matrix) concentrations. The Normalized Ar Index (NAI) and Matrix-Ar Index (MA) are used to quantify MC-ICP-MS plasma conditions and plasma mass loading, respectively. Our results show that the effect of matrix elements on IMF is largely linked to plasma conditions (i.e. NAI) and behaves as a linear function of mass loading (i.e. MA). Accordingly, the matrix effects can be significantly attenuated by increasing the NAI thereby minimising the risk of plasma "over-loading". The improved understanding of the behaviour of the matrix-induced IMF allows us to define a matrix tolerance plasma state for barium isotope analysis. The accuracy of this recommended method is further assessed by analyses of two well-studied reference materials, the SAFe D2 and the JCp-1. We expect that the analytical protocol described in this study is applicable not only to barium isotope analysis, but also to a wide range of other stable isotope measurements with MC-ICP-MS. 